\title{
Retrogression in testicular seminoma with viable metastases
}

\author{
J. G. AZZOPARDI AND A. V. HOFFBRAND
}

From the Department of Morbid Anatomy, Postgraduate Medical School of London

SYNOPSIS Stages in the retrogression of testicular seminoma are described. Eosinophilic necrosis fringed by palisaded histiocytes may be followed by fibrous replacement. Oxidation of unsaturated phospholipids in necrotic tumour may lead to deposition of lipofuscin around the lesion. Search for partially or completely scarred lesions is essential before contemplating a diagnosis of primary retroperitoneal seminoma.

Retrogressed seminoma can often be distinguished from retrogressed teratoid tumours. Apparently paradoxical teratoid metastases in association with a testicular seminoma are explained on the basis of misinterpreted retrogressed teratoid tumours in association with the seminoma.

Inguinal node metastases from testicular seminoma may be the result of abnormal lymphatic drainage following previous scrotal operations, testicular torsion etc.

Testicular scars representing a fibrosed source of origin of extragenital chorioncarcinoma and other teratomatous metastases have been recognized since Prym's (1927) description. Stowell, Sachs, and Russell (1945), however, when reporting one of the most thoroughly documented instances of primary extragenital chorioncarcinoma, disputed the significance of these testicular scars, and Lynch and Blewett (1953) went so far as to suggest that the scars themselves might be the consequence of primary extragenital growths. The speculative view of the latter workers has been quoted by others (e.g., Ainsworth and Gresham, 1960). Azzopardi, Mostofi, and Theiss (1961), on the basis of 17 cases in the files of the Armed Forces Institute of Pathology, Washington, D.C., strongly supported the contention that testicular scars can represent sites of fibrosed primary gonadal tumours and drew attention to the significance of haematoxyphil deposits in the seminiferous tubules in retrogressed testicular teratoid neoplasms. They cautioned against an uncritical acceptance of cases of allegedly primary extragenital, and especially retroperitoneal, embryonal carcinoma and chorioncarcinoma. That retrogression with scarring occurs also in testicular seminoma is not generally appreciated.

The purpose of this paper is to report a case illustrating the pattern of regression in a testicular seminoma accompanied by viable metastases and to discuss the implications of these findings.

Received for publication 19 June 1964.

3

\section{CASE HISTORY}

E.H., a man aged 45, a civil engineer, noticed swelling of the left leg three days before admission to hospital in July 1963. The leg throbbed and gradually became more painful. On the night before admission he had an episode of shortness of breath lasting 10 minutes. The only relevant past history was painful swelling of the left testis in 1961 which, according to the patient, was caused by 'orchitis': this was said to have been treated by manipulation after which the pain subsided. In view of this and the subsequent findings, this might well have been a testicular torsion.

On examination, the patient was a well-built man with a mild pyrexia up to $100^{\circ} \mathrm{F}$. and a rapid, regular pulse; his blood pressure was $120 / 70 \mathrm{~mm} . \mathrm{Hg}$, he had a normal jugular venous pressure, and no abnormalities were found in the cardio-respiratory systems. Abdominal and rectal examination was normal. No abnormal neurological signs were present. The left leg was swollen, red and hot. Peripheral pulses were palpable on both sides. In the left inguinal region there was a mass of enlarged lymph nodes which were not tender. The left spermatic cord was thickened and the epididymis felt harder than its opposite number. The left testis felt normal but was possibly slightly reduced in size. No abnormality of the right scrotal contents was detected. He was thought to have a deep vein thrombosis in the left leg due to femoral vein pressure associated with the mass in the left groin. Tuberculosis and neoplasm were considered as potential causes of the genital lesion.

Haemoglobin was 13.2 g./100 ml., white blood count $15,000 /$ c.mm. with $84 \%$ polymorphs, E.S.R. (Westergren) $23 \mathrm{~mm}$./hour. The blood urea level was $24 \mathrm{mg}$. \%, and the Wassermann and Kahn reactions were negative. No acid135 
fast bacilli were seen in the urine and no tubercle bacilli found on culture. A chest radiograph was normal, as was an E.C.G.

The patient was treated with bed rest, anticoagulants, and antibiotics; the pain and swelling in the leg diminished. On 20 August an excision biopsy of an inguinal lymph node was carried out and, as the left cord was thickened and hard, a left orchidectomy was performed with removal of the spermatic cord. He made a good post-operative recovery and was discharged on 30 August. On 16 September the left leg was found to be swollen again and the left inguinal nodes were palpable. In view of the pathological findings (see below) he was given a course of radiotherapy on a Cobalt machine, treating the left groin and iliac fossa with an incident dose of $6,000 \mathrm{r}$ over 24 days; the lumbar region received an incident dose of 3,600 $\mathrm{r}$ through an anterior field over the same period. When seen on 21 December 1963 he had improved and was feeling well.

\section{PATHOLOGY}

INGUINAL LYMPH NODE This was received in two fragments, the larger measuring $2.5 \times 1.5 \times 1.0 \mathrm{~cm}$. The node is largely replaced by metastatic seminoma (Figs. 1 and 2). There is a histiocytic granulomatous reaction in the stroma of the type frequently associated with seminoma. Staining with Best's carmine reveals abundant glycogen in the tumour cells, a feature of the classical variety of seminoma.

ORCHIDECTOMY SPECIMEN This consists of the left testis, $4 \times 2.5 \times 2.5 \mathrm{~cm}$., and the spermatic cord, $6 \times 1.5 \times 1.0 \mathrm{~cm}$. There is fibrous thickening of the tunica vaginalis. On section the testis is firm, fibrous and white. On its posterior aspect, slightly to one side of the rete, there is a group of circular, yellow amorphous areas up to $0.5 \mathrm{~cm}$. diameter and together involving an area $1.4 \times 0.8 \mathrm{~cm}$. On serial section of this zone it measures 2 to $3 \mathrm{~mm}$. in depth.

Numerous blocks show fibrosis and hyalinization of the testicular parenchyma with only occasional, barely visible ghosts of seminiferous tubules. The epididymis shows slight tubular atrophy and moderate interstitial fibrosis with occasional lymphoid foci; it is firmly bound to the testis. The caput is relatively well preserved as is the appendix epididymis. There is dense fibrosis around the vas deferens.

The most significant findings are in the block containing the yellow amorphous foci. In addition to fibrosis this contains irregular areas of eosinophilic necrotic tissue (Fig. 3): these foci vary in number from two to six at different levels. On closer inspection each focus is found to consist of closely aggregated, large, rounded, degenerate tumour cells which have lost their nuclear staining, a feature commonly

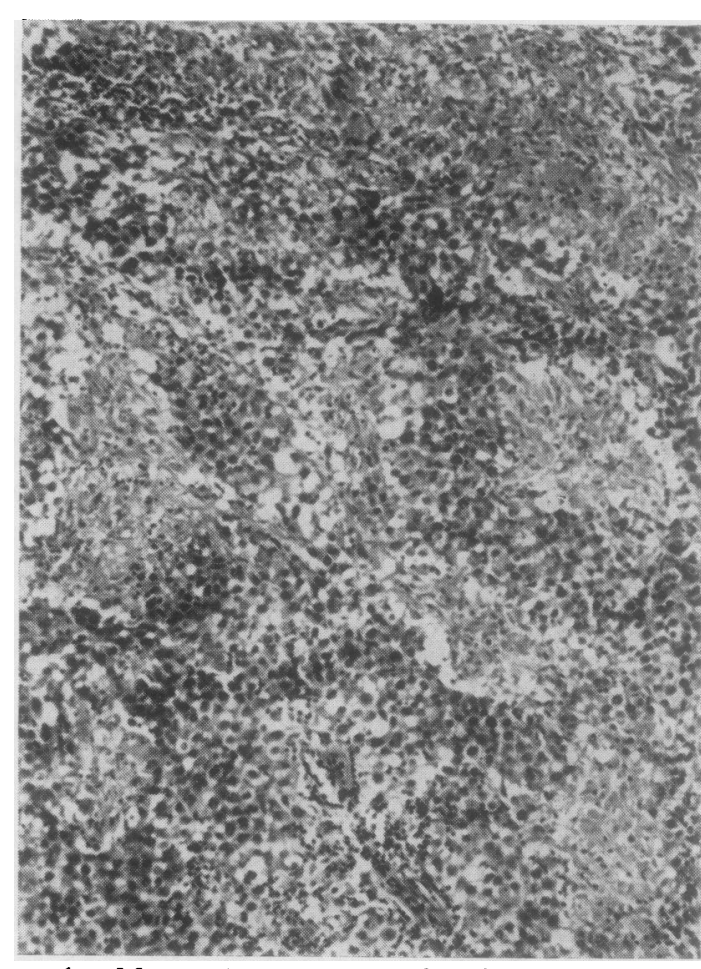

FIG. 1. Metastatic seminoma in lymph node with histiocytic stromal reaction best seen at the top. Haemalum and eosin $\times 120$.

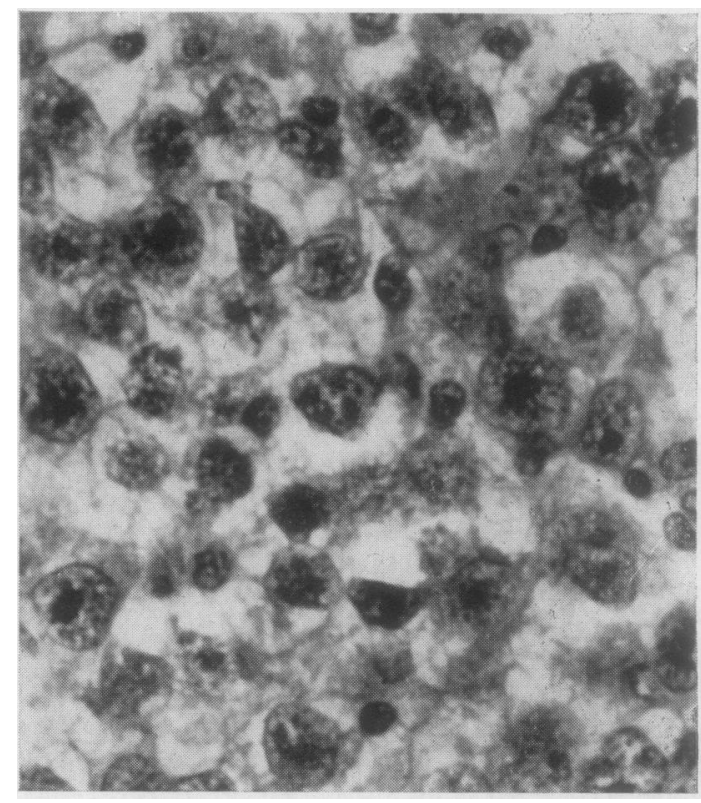

FIG. 2. Higher magnification of tumour cells in lymph

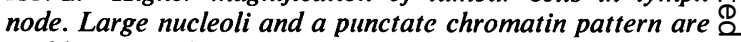
visible. Haemalum and eosin $\times 750$. 


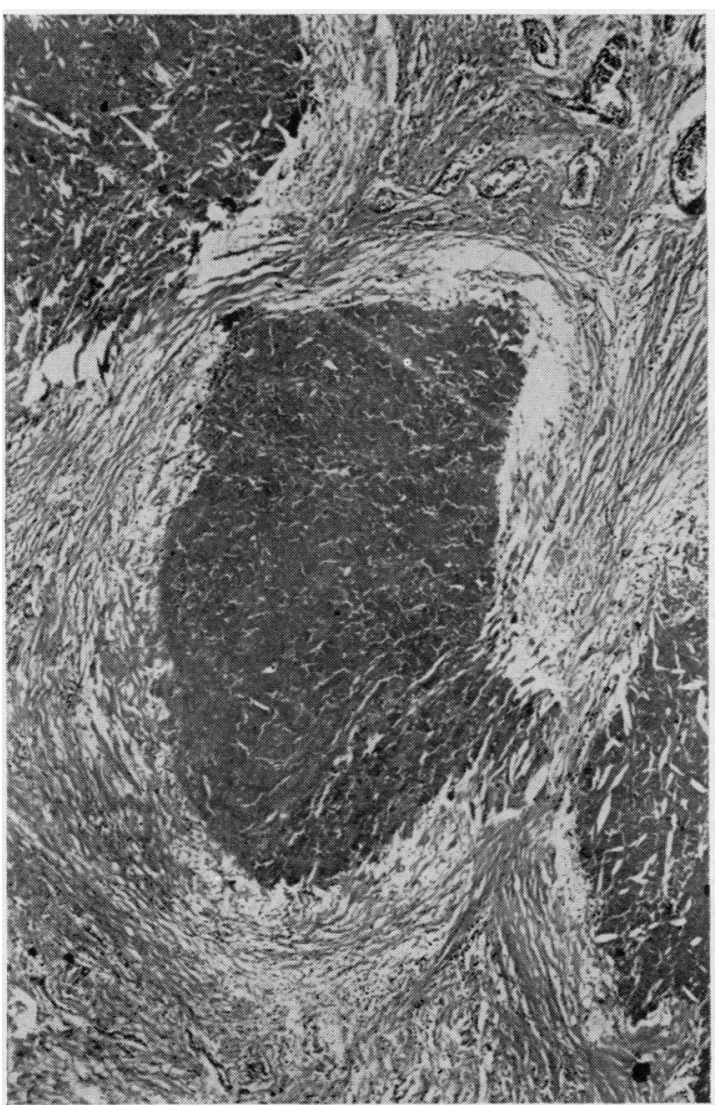

FIG. 3 .

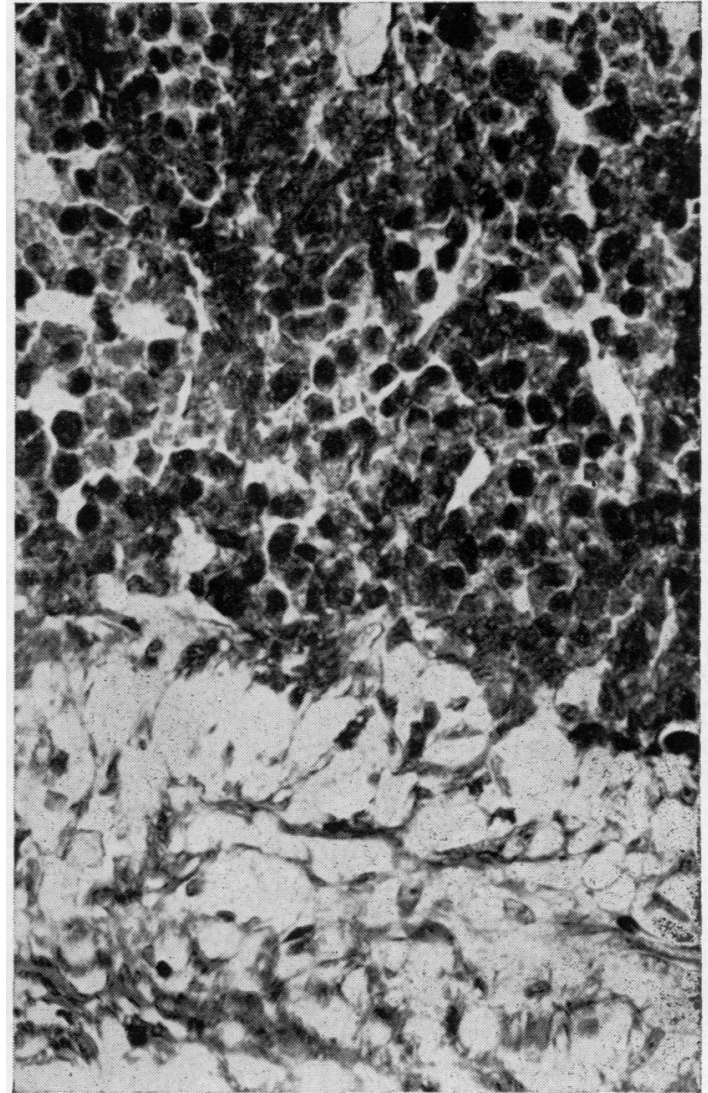

FIG. 4.

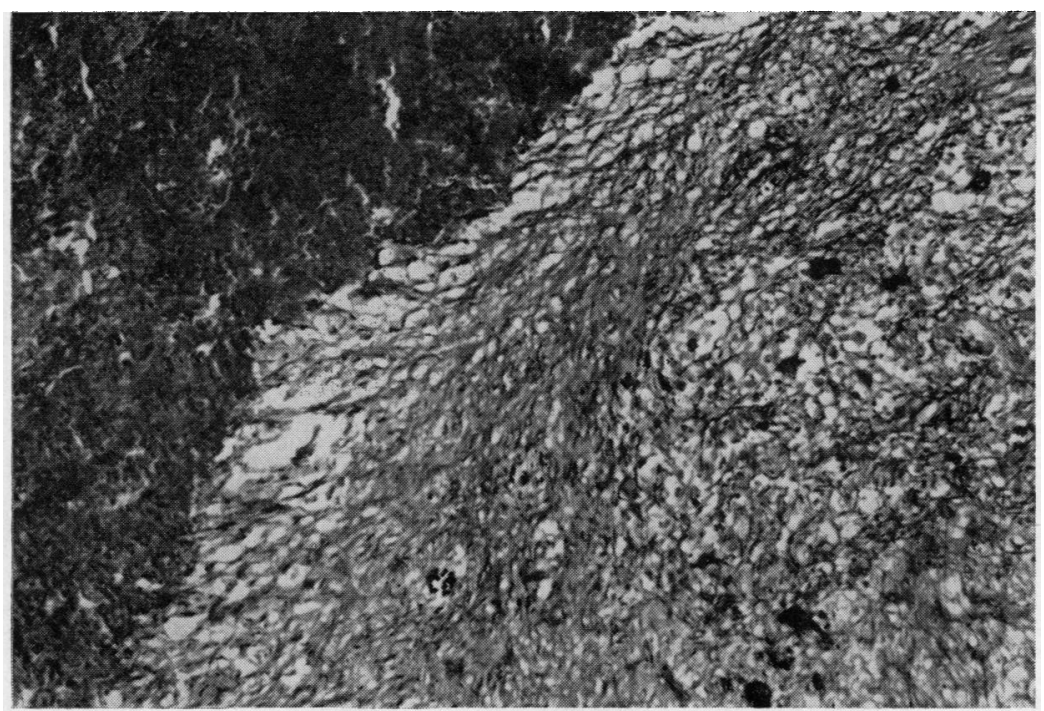

FIG. 3. Foci of eosinophilic necrotic tissue containing clefts of cholesterol esters. Calcification has resulted in the artefactual line across the photograph. Haemalum and eosin $\times 38$.

FIG. 4. Edge of necrotic focus showing outlines of individual degenerate tumour cells. Masson trichrome $\times 370$.

FIG. 5. Palisade of histiocytes and fibroblasts at edge of necrotic seminoma. Heidenhain's azocarmine $\times 100$.

FIG. 5 . 
seen in the necrotic areas of seminoma. The obscured cytological details are rendered a little clearer by staining with Masson's trichrome (Fig. 4). Serial sections, staining every fourth slide, show no evidence of viable seminoma. The necrotic foci have sharp edges and are ringed by a layer of histiocytes and fibroblasts which tend to a perpendicular orientation (Fig. 5), the whole appearance at a low magnification bearing a superficial resemblance to a subcutaneous rheumatoid nodule (Fig. 3). Occasional foamy macrophages, lymphocytes, and a few plasma cells complete the composition of the cellular infiltrate. Many of the arteries around the necrotic foci show marked intimal fibrous thickening.

In paraffin sections, the necrotic tissue stains moderately deeply with Sudan black B and is negative with P.A.S.: this indicates its phospholipid content. Since a small amount of phospholipid is present in the viable metastatic seminoma, the phospholipid at the site of the primary tumour may represent a concentration of intrinsic phospholipid by removal of protein, glycogen, and other more soluble constituents. It is not possible, however, to exclude the addition of phospholipid to the necrotic material from extraneous sources.

Haematoxyphilic deposits are present in large masses in the centre and in smaller aggregates at the edges of the necrotic foci. The Feulgen reaction is negative; the absence of deoxyribonucleic acid is not surprising in view of the ease with which it appears to be removed from necrotic seminomatous tissue. Calcium carbonate or phosphate is present in the haematoxyphilic deposits as demonstrated by naphthochrome green $B$ and Von Kossa; these stains also show a fine sprinkling of calcium salts in the necrotic tissue as a whole. A haematoxyphilic reaction has often been erroneously attributed to calcium salts when it is in fact caused by substances laid down before or together with calcium (Azzopardi, 1959). In the present case large amounts of mucopolysaccharide are present in the haematoxyphil deposits as shown by Southgate mucicarmine and by Alcian green, provided nuclear counterstains are omitted in both methods so as not to obscure the red or green staining. A small amount of greenish-yellow pigment, probably haematoidin, is present in one necrotic zone.

The histiocytes bordering the necrotic foci (Fig. 6) give a strongly positive Sudan black reaction and a positive P.A.S. reaction. On close inspection of the haemalum-eosin sections, these cells are found to contain a finely granular, pale yellow pigment. This contains no stainable iron and the suspicion that it might be lipofuscin is confirmed by the goldenyellow autofluorescence in ultra-violet light. The pigment is also acid-fast and identifiable as lipo-

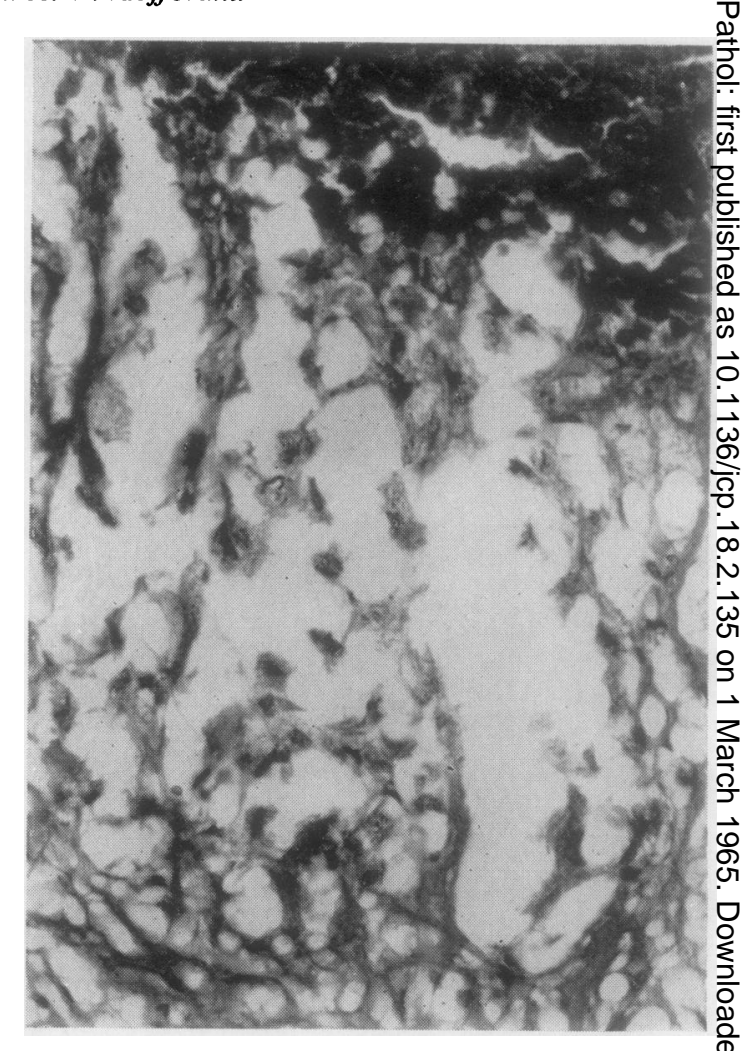

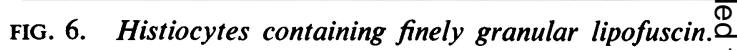
Haemalum and eosin $\times 370$.

fuscin of ceroid type. A very small amount of haemosiderin is present in histiocytes adjacent to one of the necrotic foci.

\section{DISCUSSION}

This case illustrates the stages in the regression of a primary testicular seminoma. Necrosis of tumour results in accumulations of ghosts of neoplastic cells in which cytological detail is lost and specific nuclear staining no longer possible. The degenerated tissue excites a histiocytic reaction at the periphery. $\mathbb{N}$ Cholesterol and its esters may appear in the necrotic N tumour and foam cells may be seen in the peripheral reactive zone. More important is the presence of ${ }^{\omega}$ phospholipid in the necrotic tumour; auto-oxidation? of unsaturated fatty acids of phospholipids leads to 0 the appearance of a lipofuscin pigment, of ceroid type, in the histiocytes fringing the necrosis. In the absence of specific staining, this pigment is easily mistaken for haemosiderin. Beyond the histiocytic $\vec{\mathbb{}}$ zone there is a band of dense fibrosis. In some $\frac{?}{8}$ instances, possibly because of the large quantity of $\stackrel{\mathbb{Q}}{2}$ necrotic tissue and its high fat content, necrotic? 
tumour may remain for long periods of time and become calcified. In other cases necrotic tumour may be completely removed with loss of the more specific features and replacement by a fibrous scar of variable size. In the case reported here, it is possible that the episode of probable testicular torsion suffered by the patient at an earlier date may have been an important factor in precipitating tumour necrosis. It must, however, be stressed that torsion is by no means an essential prelude to regression of testicular seminoma. In other cases seen by one of us and summarized in Table I, there was no indication of torsion in the clinical histories and in these patients the scarring and necrosis did not involve the entire testis but was limited to a part of the gonad. While radiotherapy might possibly have contributed to the scarring of the testicular lesions in some of these patients, it certainly was not responsible in cases 4 and 6 who had palliative treatment only.

Because of these findings, great caution is necessary before making a diagnosis of primary

\section{TABLE I}

\section{SUMMARY OF OTHER CASES}

$\begin{array}{lll}\begin{array}{l}\text { Case Age (yr.) } \\ \text { No. }\end{array} & \begin{array}{l}\text { Clinical Presentation } \\ \text { and Findings }\end{array} & \begin{array}{l}\text { Operative or Necropsy Macroscopic Finding } \\ \text { Findings, Progress and in Testis } \\ \text { Therapy }\end{array}\end{array}$

$142 \quad$ Pain left flank with Massive retroperitoneal Large dense scar
radiation to groin and node involvement; affecting about $2 / 3$ of testis. I.V.P. showed L. nephrectomy and cut surface. Specimen gross L. hydronephrosis nodal excision; radiotherapy. $\Delta$ ? metastatic seminoma.

Back pain, loss of central abdomen, enlarged Virchow lymph node later; orchidectomy nitrogen mustard

therapy; further weight, painless mass
L. orchidectomy.

Biopsy: seminoma. Scar, $2 \mathrm{~cm}$. max. diam., Radiotherapy to retro- involving mediastinum peritoneal mass; indurated area

L. testis 3 months

\section{Histology of Testis Additional Data and and Metastases Comment}

(a) No special features in testis scar nodes and peri-ureteric tissue

(a) At one end of scar, necrotic focus with acicular clefts, palisaded by foamy histiozytes. Pigmented histiocytes +

Some calcification

(b) Seminoma in lymph nodes

Biopsy node: seminoma. Fibrous nodule, $0.3 \mathrm{~cm}$. (a) No special features Radiotherapy to diam., near in testis scar mediastinal mass; mediastinum L. testis (b) Seminoma in metastases

courses radiotherapy and mustard. Necropsy: massive mediastinal and retroperitoneal tumour, metastases in liver,

R. adrenal, calvarium, vertebrae

Necropsy: metastases in Wedge of firm white

Low back pain,
Only palliative therapy para-aortic nodes, L. lower quadrant abdominal pain, fever, cough, loss of weight. Chest radiograph:

bilateral lung

lungs, liver, pericardium

metastases

tissue, $2 \times 2 \mathrm{~cm}$., in L. testis, equidistant between the 2 poles and
subcapsular in position

scar. Pigmented

section, minute focus of intratubular seminoma with some extratubular tumour cells. ' $G$ ' cells $+t^{1}$ (b) Seminoma with abundant necrosis in metastases

$5 \quad 31 \quad$ Anorexia and weight loss, visible swelling in L. upper abdominal quadrant

Unknown Anorexia and weight loss, low back pain, swelling L. neck, hard mass to $\mathbf{R}$. of umbilicus, mass in $\mathbf{R}$. inguinal region. Chest radiograph: mediastinal mass
Nitrogen mustard therapy. Necropsy: mass in region of duodenum, para-aortic and hepatic metastases Necropsy: metastases lungs, liver, nodes, etc.

,

.

L. testis mostly fibrous
with a small residue of recognizable parenchyma

Ill-circumscribed greyish mass replacing upper $2 / 3$ of $R$. testis and merging with residual parenchyma (a) Densely fibrous,

poorly defined scar.

' $G$ ' cells +

(b) Seminoma in

metastases

(a) Dense fibrous scar. Only palliative

Necrotic focus with therapy

'ghost' cells, acicular clefts and some D.N.A.

debris. Pigmented

histiocytes +

(b) Seminoma in

${ }^{1} \cdot G$ ' cells is an abbreviation for the altered hyperchromatic germ cells, probably spermatogonia, found within seminiferous tubules and possibly representing the earliest seminoma in situ. They are more fully described in the paper by Azzopardi et al. (1961). 
retroperitoneal seminoma. One cannot postulate origin in a third testis (Auvert, 1960) unless the testes are serially sliced and carefully examined. These difficulties apply also to primary extragenital chorioncarcinoma (Azzopardi et al., 1961). This is not to be taken as implying that primary extragenital chorioncarcinoma in the male does not occur, since there are several well-documented accounts of primary tumours of this nature arising in the mediastinum (Laipply and Shipley, 1945; Lynch and Blewett, 1953) and in the pineal region. Seminoma may also originate in the anterior mediastinum.

The testicular lesion in this case illustrates the difference between regression and healing of seminoma and regression in the teratoid group of tumours. Thirteen of 17 teratoid tumours of the testis (Azzopardi et al., 1961) contained haematoxyphil deposits in the scar tissue; these formed sharply circumscribed foci because of their localization within seminiferous tubules and were characterized by their high D.N.A. content (Fig. 7). Necrosis in

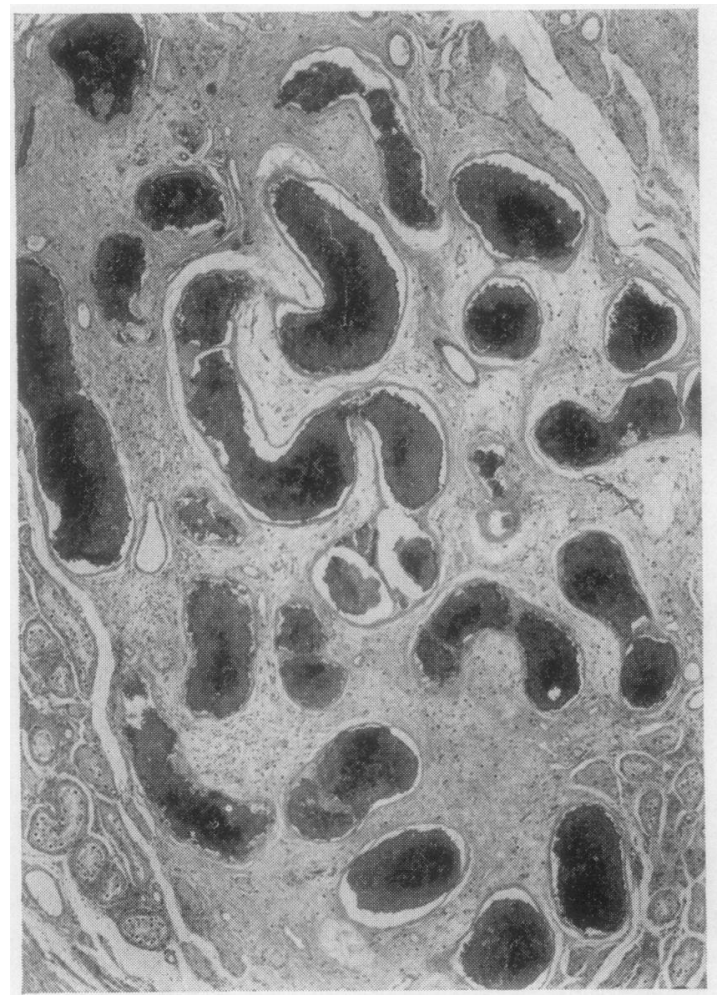

FIG. 7. Retrogression in teratoid testicular tumour. Purplish granular debris occupies distended seminiferous tubules situated in fibrous scar. This was associated with chorioncarcinomatous metastases. Haemalum and eosin $\times 38$. seminoma is mainly a feature of extratubular tumour and discrete haematoxyphil deposits are not seen. Previous authors have drawn no distinction between retrogression in seminoma and in the teratoid neoplasms of the testis. If only a fibrous scar is present in the testis, the nature of the primary tumour can only be surmised from the character of the metastases. In the presence of intratubular haematoxyphil deposits, one is justified in diagnosing a retrogressed teratoid tumour. Ghost neoplastic cells ringed by palisaded histiocytes sometimes containing lipofuscin are characteristic of the pattern of regression in seminoma, but this appearance is not specific and can be seen occasionally in other tumours. Nevertheless, where the testis contains conglomerates of 'shadow cells' associated with a histiocytic reaction we can, in the presence of seminomatous metastases, fairly assume the existence of a primary testicular seminoma. This view is supported by the present case report, by the other cases (Table I) one of us has had the opportunity of studying, and by analysis of a few cases in the literature. Quénu's (1960) patient had a fibrosed primary seminoma and cases 2 and 3 of Rather, Gardiner, and Frerichs (1954) are probably of this nature. Friedman (1951) recognized the occurrence of healing in testicular tumours. His Figs. 18 and 19, showing necrosis and sclerosis in an unspecified type of testicular tumour, very probably represent a seminoma: there is even a suggestion of palisaded histiocytes around the lesion.

The distinction between the two forms of regression has important applications and leads us to disagree with one of Friedman's fundamental conclusions. Friedman believes that the seminoma is the precursor of the embryonal carcinoma. His main evidence is that embryonal carcinomatous and chorionepitheliomatous metastases may appear when the primary testicular tumour is a 'germinoma', i.e., seminoma. Four germinomas with nongerminomatous metastases studied by the multipleblock method were found by Friedman to be pure germinomas, a finding which might tempt one to the conclusion he draws. However, if one studies his Fig. 22 it shows a good example of a seminoma alongside a retrogressed teratoma and it is lesions of the latter type that are responsible for the apparently paradoxical character of the metastases. The recognition of differences between the two types of regressed testicular tumour has histogenetic implications since it removes one of the main planks for the erroneous belief that seminoma may show transition to the teratomatous group of tumours.

A point of surgical interest in this patient, not related to the main theme, is the unusual development of and presentation with inguinal metastases 
from a testicular seminoma. Bowles (1962) has suggested that where there has been a previous scrotal operation, e.g., on a varicocele, or orchiopexy, a testicular tumour may metastasize to inguinal nodes because of alterations in the lymphatic drainage. Witus, Sloss, and Valk (1959) reported on two patients with testicular tumours developing after orchiopexy that metastasized to inguinal nodes and did not involve the retroperitoneal nodes. In the case reported here, there was an episode of probable torsion two years before the last hospital admission. Whatever the precise nature of this episode may have been, at orchidectomy the tunica vaginalis was partly obliterated by firm adhesions; this may well have resulted in variations in the normal drainage mechanism that led to the development of inguinal metastases. This case offers some support for Bowles' view of the circumstances in which testicular seminoma may involve the inguinal before the lumbar nodes. This hypothesis needs confirmation on a large series of testicular tumours.
We are grateful to Mr. A. G. Young and Dr. K. A. Misch of the Lister Hospital, Hitchin, for the use of their clinical data and pathological material, and to Dr. G. Picciotto of Mount Vernon Hospital, Northwood, for details of post-operative treatment. We wish to thank Professor C. V. Harrison and Dr. A. G. E. Pearse for their criticism, Mr. B. Chalk for technical assistance, and Miss S. Lee for typing the manuscript.

\section{REFERENCES}

Ainsworth, R. W., and Gresham, G. A. (1960). J. Path. Bact., 79, 185. Auvert, M. J. (1960). J. Urol. méd. chir., 66, 800.

Azzopardi, J. G. (1959). J. Path. Bact., 78, 513.

Mostofi, F. K., and Theiss, E. A. (1961). Amer. J. Path., 38, 207.

Bowles, W. T. (1962). J. Urol. (Baltimore), 88, 266.

Friedman, N. B. (1951). Cancer (Philad.), 4, 265.

Laipply, T. C., and Shipley, R. A. (1945). Amer. J. Path., 21, 921.

Lynch, M. J. G., and Blewett, G. L. (1953). Thorax, 8, 157.

Prym, P. (1927). Virchows Arch. path. Anat., 265, 239.

Quénu, L. (1960). J. Urol. méd. chir., 66, 861.

Rather, L. J., Gardiner, W. R., and Frerichs, J. B. (1954). Stanf. med. Bull., 12, 12.

Stowell, R. E., Sachs, E., and Russell, W. O. (1945). Amer. J. Path., 21, 787.

Witus, W. S., Sloss, J. H., and Valk, W. L. (1959). J. Urol. (Baltimore), 81,669 . 